\title{
Coiled Coil-Mediated Assembly of an Icosahedral Protein Cage with Extremely High Thermal and Chemical Stability
}

\author{
Ajitha S. Cristie-David ${ }^{a}$, Junjie Chen ${ }^{\mathrm{a}}$, Derek B. Nowak ${ }^{\mathrm{b}}$, Sung I. Park ${ }^{\mathrm{b}}$, Mark M. Banaszak \\ Holl $^{\mathrm{a}}$, Min $\mathrm{Su}^{\mathrm{c}}$ and E. Neil G. Marsh ${ }^{\mathrm{a}, \mathrm{d}, 1}$
}

a Department of Chemistry, University of Michigan, Ann Arbor 48109

${ }^{b}$ Molecular Vista Inc, Via Del Oro Suite 110, San Jose, CA 95119

${ }^{c}$ Life Sciences Institute, University of Michigan, Ann Arbor 48109

${ }^{d}$ Department of Biological Chemistry, University of Michigan, Ann Arbor 48109

\author{
${ }^{1}$ To whom correspondence should be addressed: \\ Dr. Neil Marsh \\ nmarsh@umich.edu \\ Department of Chemistry \\ University of Michigan \\ Ann Arbor MI 48109 \\ Phone 7347636096
}

Keywords: hyper-stable proteins; protein design; atomic force microscopy; photo-induced force microscopy; cryo-electron microscopy 


\begin{abstract}
The organization of protein molecules into higher-order nanoscale architectures is ubiquitous in Nature and represents an important goal in synthetic biology. Here we describe the symmetrydirected design of a hollow protein cage with dimensions similar to those of many icosahedral viruses. The cage was constructed based on icosahedral symmetry by genetically fusing a trimeric protein (TriEst) to a small pentameric de novo-designed coiled coil domain, separated by a flexible oligo-glycine linker sequence. Screening a small library of designs in which the linker length varied from 2 to 12 residues identified a construct containing 8 glycine residues (Ico8) that formed well-defined cages. Characterization by dynamic light scattering, negative stain and cryo EM, and by atomic force and IR-photo-induced force microscopy established that Ico8 assembles into a flexible hollow cage with comprising 60-subunits with overall icosahedral geometry. Unexpectedly, the cages were found to encapsulate DNA, even though neither protein component binds nucleic acids on its own. Notably, the cages formed by Ico8 proved to be extremely stable towards thermal and chemical denaturation: whereas TriEst was unfolded by heating $\left(\mathrm{T}_{\mathrm{m}} \sim 75^{\circ} \mathrm{C}\right)$ or denatured by $1.5 \mathrm{M}$ guanidine hydrochloride, the Ico8 cages remained folded even at $120{ }^{\circ} \mathrm{C}$ or in $8 \mathrm{M}$ guanidine hydrochloride. The encapsulation of DNA and increased stability of the cages are new properties that emerge from the higher order structure of the protein cage, rather than being intrinsic to the components from which it is constructed.
\end{abstract}




\section{Introduction}

Self-assembling proteins are attractive as building blocks for "smart" biomaterials that can potentially exploit the rich structural and functional properties of proteins (1-4). This has spurred efforts to design new self-assembling protein structures by applying symmetry principles to construct geometric cages and extended lattices (5-10). Successful designs have utilized computational approaches to design new interfaces between protein subunits to facilitate assembly $(3,11-13)$, or the fusion of two oligomeric proteins through a rigid alpha-helical linker (14). Using these strategies, tetrahedral, cubic (14), octahedral (12, 13), dodecahedral and icosahedral $(3,11)$ protein cages as well as 2 - and 3 -dimensional $(15,16)$ protein lattices have been constructed. However, these approaches require that the angle between the two rotational symmetry axes that defines the geometry of the assembly needs to precisely specified for the protein subunits to assemble correctly. This has proved technically challenging to accomplish.

Our laboratory has developed an alternative design strategy, in which protein assembly is mediated by short, parallel, coiled coil domains that are genetically fused to the larger building block protein through a short, flexible spacer sequence (17-19). This approach, greatly simplifies the design process because angle between the symmetry axes does not need to specified. By using combinations of symmetry axes that are unique to the geometry of the desired protein cage, it is possible to assemble well-defined protein cages using symmetry considerations alone. Thus, we have recently described the construction of octahedral (19) and tetrahedral (17) cages by combining a $\mathrm{C}_{3}$-symmetric building block protein with either a $\mathrm{C}_{4}$ symmetic or $\mathrm{C}_{3}$-symmetric coiled coil assembly domain, respectively. This approach is inherently modular, as many coiled designs are available and there are few constraints on the choice of protein building block; it is also far less computationally intensive than protein interface re-design.

Here, we demonstrate the extension of this approach to the design of an icosahedral cage. As the largest Platonic solid, icosahedral protein cages can encapsulate large volumes and as such are often used to in Nature for packaging and transport (20). Large, robust and customizable protein cages should be useful in synthetic biology $(1,21)$, vaccine design (22) and targeted drug delivery (23). However, icosahedral cages remain challenging to construct, as they require the coordinated assembly of 60 protein subunits to achieve the desired icosahedral geometry. 
For the combination of $\mathrm{C}_{3}$ and $\mathrm{C}_{5}$ symmetric proteins necessary to specify an icosahedral cage, we selected a trimeric esterase (TriEst) as the protein building block and a wellcharacterized pentameric parallel coiled-coil domain. These were linked together through a flexible oligo-Gly sequence. Characterization of the resulting protein assemblies revealed that they form hollow cages, with features consistent with the intended 60-subunit icosahedral geometry. Notably, assembly of the trimeric esterase into a cage resulted in a remarkable increase in both its thermal stability and resistance to chemical denaturation by guanidium hydrochloride $(\mathrm{GuHCl})$ and extremes of $\mathrm{pH}$.

\section{Results}

To design a self-assembling icosahedral protein cage, we followed the approach previously taken to design tetrahedral and octahedral cages. For the $\mathrm{C}_{3}$-symmetric building block we used the same trimeric esterase (PDB ID: 1ZOI) as in our previous studies to facilitate comparisons with our earlier designs and assess the generality of the design strategy (24). To provide the $\mathrm{C}_{5}$ component necessary for icosahedral geometry we selected a de novo-designed, parallel, pentameric coiled coil (25) (PDB ID: 4PN8); this component was modified by mutating W13Q to alleviate problems with aggregation, as described previously (18).

\section{Screening and Optimization of Assembly}

Synthetic genes were constructed to encode proteins in which the C-terminus of TriEst was linked to the $\mathrm{N}$-terminus of the coiled coil through a flexible sequence containing increasing numbers of Gly residues. (Fig. 1 \& Table S1). The number of Gly residues were increased two at a time to give constructs containing between 2 and 12 Gly, herein referred to as Ico2 through Ico12 respectively, with each additional di-Gly unit increasing by $\sim 5 \AA$ the distance between the $\mathrm{C}_{3}$ and $\mathrm{C}_{5}$ domains. These constructs were expressed and purified by standard procedures as described in the methods section.

Preliminary characterization of Ico2 and Ico4 indicated that the proteins were unstable and rapidly precipitated, presumable because the linker length was too short for the protein domains to fold properly. The other constructs were sufficiently stable to be characterized by SEC and negative stain EM. Ico6, Ico10 and Ico12 each formed a heterogeneous mixture of assembled proteins, aggregated material and unassembled trimers (Fig. S1). However, Ico8 assembled into spherically shaped assemblies that were of approximately the size expected for a 
60 -subunit protein cage (estimated to be $\sim 27 \mathrm{~nm}$ in diameter), as determined by size exclusion column chromatography (SEC), dynamic light scattering and negative stain EM (Fig 2). The size distribution, estimated from analysis of negative stain EM images of the particles was quite broad, ranging from $\sim 24-30 \mathrm{~nm}$ in diameter. These findings were consistent with the flexible nature of the design, as the oligo-Gly spacer sequence has the potential to span a length of $\sim 20$ $\AA$. However, we don't rule out the possibility that some particles may have misassembled and contain either fewer or greater than the intended 60 protein subunits. Higher resolution images were obtained using scanning transmission electron microscopy (STEM) (Fig. 2C). These images revealed hexagonal features in many of the particles, particularly apparent in the Fourierfiltered images, which is consistent with the intended icosahedral geometry of the protein cages.

Based on these initial studies, the protein cages formed by Ico8 were selected for further detailed characterization. Although Ico8 contained an N-terminal His tag, the protein bound only weakly to Ni-NTA resin used in the initial purification. We therefore took advantage of the large size and heat stability of the assembled protein (discussed later) to purify Ico8. This was achieved by heating the cell lysate at $70{ }^{\circ} \mathrm{C}$ for $\sim 75 \mathrm{~min}$ to precipitate most E. coli proteins, which were removed by centrifugation. After dialysis, the cages were purified further by two rounds of SEC. This procedure produced electrophoretically pure material (Fig. S1) that was used for further characterization.

\section{Atomic force microscopy of Ico8}

To obtain further information on the size of the cages formed by Ico8, we imaged the cages using atomic force microscopy (AFM, Fig. 3A), which has the advantage of directly measuring the height and volume of particles. Particles were spin-coated onto a mica substrate as described in the Methods section and imaged using a carbon nanotube (CNT) tip in AC mode. 1100 particles were imaged and subjected to automated particle analysis and classification (Fig 3B, Fig S2). Consistent with their flexible, hollow nature, the cages flattened onto the freshly cleaved mica surface resulting in an average particle height of $3.6 \pm 1.1 \mathrm{~nm}$. To compare to the theoretically expected volume and the experimental volume obtained from cryo-electron microscopy (cryo-EM), the measured particle volumes were used to calculate the diameter of an ideal icosahedral geometry. This yielded an average diameter of $24.9 \pm 7.0 \mathrm{~nm}$ for the Ico8 cages, with the distribution of particle sizes being in good agreement with that measured by both cryo-EM and negative stain EM (vida infra). 
Further images and Infrared (IR) spectra for individual Ico8 particles were measured using IR-photo-induced force microscopy IR-PiFM (Fig. 3C,D). This technique records the vibrational spectrum of the material located in close proximity of an AFM tip operated in dynamic mode $(26,27)$. IR spectra recorded from individual particles showed characteristic amide-I and amide-II bands, confirming that the particles consist of proteins (Fig. 3D). Images obtained using the amide I band at $1666 \mathrm{~cm}^{-1}$ exhibited facets associated with the particles that are consistent with the formation of icosahedral cages. Interestingly, the particles appeared spherical under conventional AFM analysis, even when using a CNT tip.

\section{Cryo-electron microscopy of Ico8}

Further structural information on the cages formed by Ico8, was obtained by cryo-EM single particle analysis. A total of 18,707 particles were excised then subjected to reference-free classification and averaging using the program RELION (28). 2D class-averaged cryo-EM images (Fig. S3) indicate Ico8 forms hollow cages that range in diameter from 20 to $30 \mathrm{~nm}$, with an average diameter $\sim 25 \mathrm{~nm}$ (Fig. 4A,B). Unexpectedly, two shells of density were observed in all the class averages, with most classes exhibiting short, inward-pointing features that appear to connect the inner and outer shells at multiple sites. The thickness and gap between the two shells appeared independent of the sizes of these cages (Fig S4). Interestingly, these inward-pointing features seem compatible with the size of the pentameric coiled coil domain (Fig. 4B).

We then selected 10,363 particle projections, representing the average size of the protein cage, to calculate a low-resolution 3D cryo-EM reconstruction of Ico8 using the program cisTEM (29). Icosahedral symmetry was enforced during the refinement and the final reconstruction indicated a resolution of $12 \AA$. The symmetrically reconstructed electron density map clearly reveals the arrangement of esterase trimers that surround a region of inward-pointing density attributable to the 5-fold symmetric coiled coil domain (Figure 4C-E). This is consistent with inward-pointing density seen in the 2D-class averages. The orientation of the coiled coil, inward or outward, was not specified in the design and it is possible that there is a minor population of coiled coils that point outwards but are not resolved by cryo-EM. The inner shell of electron density is also clearly evident in the reconstruction.

It is evident that the electron density poorly accommodates the structure of TriEst, as the density is thinner and more spread out than expected (Fig. 4C). This is attributable to the flexible nature of the protein cage, which leads to smearing out of the averaged density. 
However, although of low resolution, the structure could be reproducibly reconstructed from the data. Furthermore, the icosahedral features of the cage were largely maintained when the symmetry used in the reconstruction was reduced to $\mathrm{C}_{5}$, providing confidence that the icosahedral geometry is correct.

\section{Ico8 encapsulates DNA}

As noted above, we were surprised that the both $2 \mathrm{D}$ class averages and the $3 \mathrm{D}$ reconstruction identified an inner shell of strongly scattering electron density within the cages. Noting that nucleic acids scatter more strongly than proteins in cryo-EM images, and that thickness of the inner wall is similar to the diameter of a DNA double helix, we decided to investigate whether nucleic acids may be encapsulated within the cages; interestingly, this appears to be the case.

Protein cages were extracted with phenol:chloroform to remove the protein and the nucleic acids analyzed by electrophoresis, either before or after digestion with RNase A or DNase I. The recovered nucleic acids fragments were approximately $300-500 \mathrm{bp}$ in size and could be digested by DNase I treatment (but not RNase A treatment) indicating that they comprise DNA (Fig S4). Quantification of the nucleic acid and protein content of the cages, determined by agarose electrophoresis and SDS-PAGE respectively, indicated that the nucleic acid component comprises $\sim 30 \%$ by weight of the purified Ico 8 cages. We note that the cages are certainly large enough to accommodate this amount of DNA; indeed, viral capsids of similar diameters can encapsulate single-stranded genomes of $4-5 \mathrm{kbp}(30)$. Therefore, we ascribe the inner "shell" of the cages to DNA that is non-specifically associated with the inner face of the protein cage.

\section{Stability of Ico8}

Many natural protein cages, e.g. viral capsids, exhibit high stability towards unfolding. We therefore examined how assembly of the TriEst building block into an icosahedral cage affected its stability and catalytic activity. Circular dichroism (CD) was used to follow the thermally-induced unfolding of both Ico8 and TriEst by monitoring the decrease in ellipticity at $222 \mathrm{~nm}$ (Fig. 5A). Whereas TriEst irreversibly denatured between 70 and $80^{\circ} \mathrm{C}$, Ico8 remained folded at $98{ }^{\circ} \mathrm{C}$ (the highest temperature examined). The thermal stability of Ico8 was further investigated using differential scanning calorimetry (DSC), which permitted thermal unfolding of the protein to be studied at temperatures up to $120^{\circ} \mathrm{C}$ at a pressure of $3 \mathrm{~atm}$ (Fig. S5). Again, 
TriEst underwent an irreversible exothermic transition between $70{ }^{\circ} \mathrm{C}$ and $80{ }^{\circ} \mathrm{C}$, indicative of protein unfolding; however, Ico8 exhibited no thermal transitions up to $120^{\circ} \mathrm{C}$, implying that it remained folded. Consistent with this, no discernable change in morphology was apparent when the protein cages were imaged by EM after cooling (Fig. S6). The esterase building block retained full catalytic activity, determined with 2,4-dinitrophenol acetate as substrate, when assembled into Ico8 cages and, significantly, remained fully active after heating and cooling (Table S2). In contrast, as expected, TriEst was completely inactivated by heat treatment.

The cages formed by Ico8 also proved extremely stable towards chemical denaturation. We compared the stability of Ico8 and TriEst at pH 2.0 and pH 12.0. At low pH the TriEst building block was unfolded and/or aggregated. However, both CD spectroscopy and DLS measurements indicated that the cages formed by Ico8 remain intact. Similarly, Ico8 remained folded even in the presence of $8 \mathrm{M} \mathrm{GuHCl}$, whereas TriEst was irreversibly unfolded by $\sim 1.5 \mathrm{M}$ $\mathrm{GuHCl}$ (Fig. S7). We extended these measurements by using temperature-dependent DLS to examine the thermal stability of $\mathrm{Ico} 8$ at $\mathrm{pH} 2.0$, at $\mathrm{pH} 12.0$, and in $8 \mathrm{M} \mathrm{GuHCl}$ (Fig. 5B). The number-averaged diameter distribution of particles recorded by DLS provides a sensitive measure of protein aggregation, which is indicative of partial unfolding. At $\mathrm{pH} 2.0$, Ico8 showed no evidence for unfolding/aggregation at temperatures up to $98^{\circ} \mathrm{C}$. At $\mathrm{pH} 12.0$, or in $8 \mathrm{M}$ $\mathrm{GuHCl}$, an increase in particle diameter, indicative of aggregation, was only evident when the protein samples were heated to temperatures above $90{ }^{\circ} \mathrm{C}$. Again, no discernable change in morphology was apparent when the protein cages were imaged by negative stain EM after treatment with denaturants. The only exception was samples heated in $8 \mathrm{M} \mathrm{GuHCl}$, for which some aggregation was evident by EM (Fig. S6). Interestingly, when the cages heated in $\mathrm{GuHCl}$ were re-imaged after storing at room temperature for 2 weeks, the aggregated material was no longer evident although the cages appeared more heterogeneous in size. (Fig S6).

\section{Discussion}

Using a simple, symmetry-based approach, we demonstrate the assembly from "off-theshelf" components of a nano-scale protein container with a size comparable to a viral capsid. Remarkably, the combination of a $\mathrm{C}_{3}$-symmetric building protein block and a $\mathrm{C}_{5}$-symmetric coiled coil, appears to be sufficient to assemble an icosahedral protein cage. As with our previous tetrahedral and octahedral cage designs $(17,19)$, it was necessary to optimize the linker 
length to achieve efficient assembly, but this required screening only a small number of constructs to identify a successful design. Importantly, the assembly process does not compromise the catalytic activity of the enzyme, which is actually higher for Ico8 than the esterase from which Ico8 is assembled (Table S2). Thus, our design approach appears to be both an efficient and general method to assembling proteins that requires minimal computational modeling to implement. The relative ease with which tetrahedral, octahedral and icosahedral protein cages are formed suggests that natural protein cages may perhaps have evolved through a similar route involving gene fusion of $\mathrm{C}_{3}$-symmetric proteins with $\mathrm{C}_{3}$ -, $\mathrm{C}_{4}$ - or $\mathrm{C}_{5}$-symmetric proteins. If the cages conferred an advantage on the organisms in which they arose, then further rounds of natural selection may have led to the development of more rigid cages with extensive protein interfaces.

The size distribution of the Ico8 cages is broader than that seen for the octahedral and tetrahedral cages we described previously $(17,19)$. This probably reflects the flexibility of the cages that arises from the flexible 8-Gly linker connecting the TriEst and coiled coil domains and the fact this icosahedral cage is much larger than the octahedral and tetrahedral cages. This flexibility is evident in the electron density of the reconstructed structure obtained by cryo-EM, in contrast, and perhaps unsurprisingly, better-defined cryo-EM reconstructions were obtained for the smaller tetrahedral and octahedral cages assembled with coiled coils. Further evidence for Ico8 forming 60-subunit icosahedral structures comes from individual EM and PiFM images where hexagonal features consistent with icosahedral geometry are also clearly evident.

An interesting feature of the cages formed by Ico8 is their extremely high thermal and chemical stability. Whereas TriEst is irreversibly unfolded at $75^{\circ} \mathrm{C}$, and in the presence of 1.5 $\mathrm{M} \mathrm{GuHCl}$, the Ico8 cages remain intact at $120^{\circ} \mathrm{C}$ and in $8 \mathrm{M} \mathrm{GuHCl}$. This places Ico8 among some of the most thermostable proteins so far described, either natural or designed. The enzymatic activity of the cages is not affected by exposure to these harsh conditions, which is clearly a valuable feature for many biotechnological applications. An icosahedral protein cage, thermostable to $90{ }^{\circ} \mathrm{C}$, which was engineered by designing new subunit interfaces, was recently reported by Baker and co-workers (3). However, in this case the protein building block was obtained from a hyperthermophile and so was intrinsically thermostable. As shown in Fig. 5A, TriEst is not itself very thermostable, posing a question as to the origin of the remarkable stability exhibited by Ico8. This may partly derive from the stability of the coiled coil domain, 
which was previously shown to withstand thermal unfolding (25). Further, cooperative effects that arise from the increased number of inter-subunit interactions in the assembled cage, also may contribute to Ico8's stability. Similar cooperative interactions between capsid proteins are suggested to the high stability of many viral capsids (31-33). The high rigidity of viral capsids has also been cited as a source of their stability (31-33); however, our results suggest that, contrary to prevailing assumptions, protein rigidity is not a prerequisite for assembling extremely stable protein cages.

An unanticipated feature of the cages formed by Ico8 is that they appear to encapsulate nucleic acids, most likely DNA. When visualized by cryo-EM the nucleic acid appears to form a separate shell with in the protein cage. This was unexpected because neither TriEst, nor the coiled coil binds nucleic acids, and nucleic acids did not bind to either of the previously constructed octahedral or tetrahedral cages. In this respect, nucleic acid encapsulation may be considered a truly emergent property of I $\cos$ because it is not a property of the protein components and was not intentionally designed into the cage. This observation intriguingly hints at a mechanism whereby viruses could have evolved, de novo, from cellular components through the adventitious encapsulation of nucleic acids. 


\section{Materials and Methods}

\section{Construction of genes encoding fusion proteins.}

Codon-optimized genes ligated into the expression vector pET28b were either commercially synthesized or derived from the other constructs using standard techniques. The sequences of the proteins are included in Table S1.

\section{Protein expression and purification}

Expression constructs were transformed into E. coli BL21(DE3) cells and protein expression induced by IPTG using standard methods as described previously (17, 19). For initial characterization, proteins were purified by standard methods using an N-terminal 6-His tag to facilitate affinity chromatography on a Ni-NTA column as described previously $(17,19)$. For further characterization of Ico8, the protein was purified as follows: $\sim 5 \mathrm{~g}$ of cells was resuspended on ice in $45 \mathrm{~mL}$ of $50 \mathrm{mM}$ HEPES buffer, $\mathrm{pH} 7.5$ containing $300 \mathrm{mM}$ ammonium acetate, $50 \mathrm{mM}$ imidazole, $1.5 \mathrm{M}$ urea and 5\% (v/v) glycerol; a protease inhibitor tablet (benzonase) and $50 \mathrm{mg}$ of lysozyme were added and the suspension gently shaken for $20 \mathrm{~min}$. The cells were lysed by sonication and the lysate clarified by centrifugation for $30 \mathrm{~min}$ at 40,000 $g$. The supernatant was passed through a $5 \mathrm{~mL} \mathrm{Ni-NTA}$ column at $0.25 \mathrm{~mL} / \mathrm{min}$ and the flowthrough heated at $70{ }^{\circ} \mathrm{C}$ for $75 \mathrm{~min}$. Precipitated proteins were removed by centrifugation at $40,000 \mathrm{~g}$ for $30 \mathrm{~min}$ and the supernatant dialyzed at $4{ }^{\circ} \mathrm{C}$ against $20 \mathrm{mM}, \mathrm{pH} 7.5$, HEPES, $2 \mathrm{mM}$ EDTA, $100 \mathrm{mM}$ ammonium acetate for one week. Dialyzed samples were further purified by 2 rounds of SEC using a Superose-6 300/10 column equilibrated in the same buffer (flow rate 0.3 $\mathrm{mL} / \mathrm{min}$ ). Fractions containing Ico8 were pooled, concentrated by ultrafiltration and rechromatographed on the same column. Samples were then concentrated and stored at room temperature.

\section{Enzyme Activity}

The esterase activity of Ico8 and Tri-Est were assayed using 2,4-dinitrophenol acetate as the substrate and measuring the increase in absorbance at $405 \mathrm{~nm}$, as previously described (17).

\section{Nucleic acid analysis}

Nucleic acids were purified from protein samples by phenol:chloroform extraction using standard methods. The presence of DNA, rather than RNA, was confirmed by digestion with either DNAase I (RNAase free) or RNAase, followed by gel electrophoresis. $500 \mathrm{ng}$ of phenol:chloroform extracted nucleic acids were incubated with either 1 unit of DNAase I or 
RNAase A for 30 min at $25{ }^{\circ} \mathrm{C}$ in the manufacturers' buffer. The amount of DNA was quantified by analysis of agarose gels and comparison to DNA standards using the program Image J.

\section{Electron Microscopy}

Proteins $(0.03-0.1 \mathrm{mg} / \mathrm{mL})$ were fixed on Formvar/Carbon $400 \mathrm{Mesh}, \mathrm{Cu}$ grids using conventional procedures and staining with uranyl formate. Imaging was performed at room temperature with JEOL 1500 electron microscope equipped with tungsten filament, XR401 high sensitivity CMOS camera and operated at $90 \mathrm{keV}$. Samples for scanning transmission electron microscopy were prepared similarly. Images were acquired using a JEOL JEM-2100F transmission electron microscope (TEM) with a CEOS probe corrector. Microscopy was performed at $200 \mathrm{keV}$ in scanning transmission microscopy (STEM) mode with the lens setting corrected by the Cs-corrector to produce a sub-Angstrom beam size. Both high-angle annular dark-field and bright-field images were acquired simultaneously. To better display the internal microstructures of the virus, fast Fourier transform image filtering was performed for bright field images.

\section{Dynamic Light Scattering}

DLS measurements were made using a DynaPro Nanosizer ZS instrument. Measurements were made at $25{ }^{\circ} \mathrm{C}$ with the refractive index and absorption coefficient for the particles were set at 1.45 and 0.001 respectively. Diameter distributions by intensity were recorded. Measurements were made in triplicate with each measurement comprising an average of 15 scans. Temperaturedependent DLS was performed using the same instrument. $10 \mu \mathrm{l}$ samples with protein concentration $0.2 \mathrm{mg} / \mathrm{ml}$ were heated at a rate of $5{ }^{\circ} \mathrm{C} / \mathrm{min}$ from 40 to $100{ }^{\circ} \mathrm{C}$. The refractive index and absorption coefficient for the particles were set at 1.45 and 0.001 respectively, and the diameter distribution by intensity was recorded.

\section{Circular Dichroism (CD)}

CD measurements were performed using Jasco J815 CD spectrometer. Protein concentrations were between $0.5-0.2 \mathrm{mg} / \mathrm{mL},\left(1 \mathrm{~mm}\right.$ path length cuvette). Samples were heated from $40^{\circ} \mathrm{C}$ to $98{ }^{\circ} \mathrm{C}$ at a rate of $1{ }^{\circ} \mathrm{C} / \mathrm{min}$.

\section{Differential Scanning Calorimetry (DSC)}

DSC scans were performed using a TA instruments nanoDSC, with dialysis buffer was used as reference. $\sim 0.6 \mathrm{~mL}$ of samples of protein, $0.3-0.4 \mathrm{mg} / \mathrm{mL}$, were heated at $1{ }^{\circ} \mathrm{C} / \mathrm{min}$ from $50{ }^{\circ} \mathrm{C}$ 
to $115^{\circ} \mathrm{C}$ at $3 \mathrm{~atm}$ pressure and the changes in heat taken up by the samples recorded.

\section{Atomic force microscopy (AFM)}

Samples for AFM analysis were first chemically cross-linking using the lysine-specific reagent bis(sulfosuccinimidyl)suberate (Thermo Fisher) as previously described (17) to achieve $\sim 50 \%$ cross-linking as determined by SDS-PAGE. The protein solution $(20 \mu \mathrm{L} \sim 0.2 \mathrm{mg} / \mathrm{mL})$ was spincoated at $3500 \mathrm{rpm}$ onto a freshly cleaved mica surface and further rinsed with $40 \mu \mathrm{L}$ milli-Q water while spinning to remove salt from the surface. AFM imaging was carried out under AC mode using PicoPlus 5500 (Agilent Electronic Measurement, K-tek CNT tip) AFM system. A total of 20 images containing $\sim 1100$ cages were analyzed by the automated particle detection function in SPIP (version 6.2.6, Image Metrology, $0.8 \mathrm{~nm}$ z-range cutoff threshold). The diameter was calculated from the measured volume using the icosahedral geometric relationship: $V=\frac{5(3+\sqrt{5})}{12} *\left(\frac{2 d}{\sqrt{10+2 \sqrt{5}}}\right)^{3}$, where $\mathrm{V}$ is the volume and $\mathrm{d}$ is the diagonal diameter.

The IR-PiFM measurements were performed using a Molecular Vista Inc, Vista-IR microscope. A QCL based light source illuminated the AFM probe $(300 \mathrm{kHz} \mathrm{NCHR}-\mathrm{Au}$, NanoSensors AG) providing a spectral range from $770-1885 \mathrm{~cm}^{-1}$. The protein solution $(20 \mathrm{uL}$, $0.2 \mathrm{mg} / \mathrm{mL}$ ) was drop-cast onto freshly cleaved mica and air dried before imaging. The spectral acquisition time was 1.0 seconds with image acquisition time of $4.2 \mathrm{~min}$ at $256 \mathrm{x} 256$ pixels.

\section{Cryo-electron microscopy}

Samples were concentrated to $\sim 0.3 \mathrm{mg} / \mathrm{ml}$ and loaded onto glow-discharged QUANTIFOIL R2/2 200 mesh grids and flash-frozen using Vitrobot (FEI Mark IV). The samples were visualized at liquid nitrogen temperature on a Talos Arctica electron microscope (FEI) operating at $200 \mathrm{kV}$. Cryo-EM images were recorded at a nominal magnification of $\times 34014$ using a K2 Summit direct electron detector (Gatan Inc.) in counted mode.

A total of 560 micrographs were taken using automation software SerialEM (34), Dosefractionated image Images were subjected to motion correction using MotionCor2 (35), and binned by 2 resulting in a sampling of $2.94 \AA$ per pixel for particle picking and processing. 18,707 particles were selected automatically, then subject to reference-free 2D classifications using RELION (28). Well-defined class average images were selected for further 3D reconstruction using cisTEM (29). 


\section{Author contributions}

A.S.C-D., J.C., D.B.N. and M.S. performed the experiments. A.S.C-D., J.C., D.B.N., M.S., M.M.B.H. and E.N.G.M. designed the experiments. A.S.C-D., J.C., D.B.N., M.S., M.M.B.H. and E.N.G.M. analyzed the data and wrote the manuscript.

\section{Competing financial interests}

The authors declare no competing financial interests.

\section{Acknowledgements}

We thank Dr. Kai Chen, University of Michigan for help in acquiring STEM images. This work was supported in part by grants from the National Institutes of Health (GM 093088) and the Army Research Office (W911NF-11-1-0251) to E.N.G.M. 


\section{Figures}

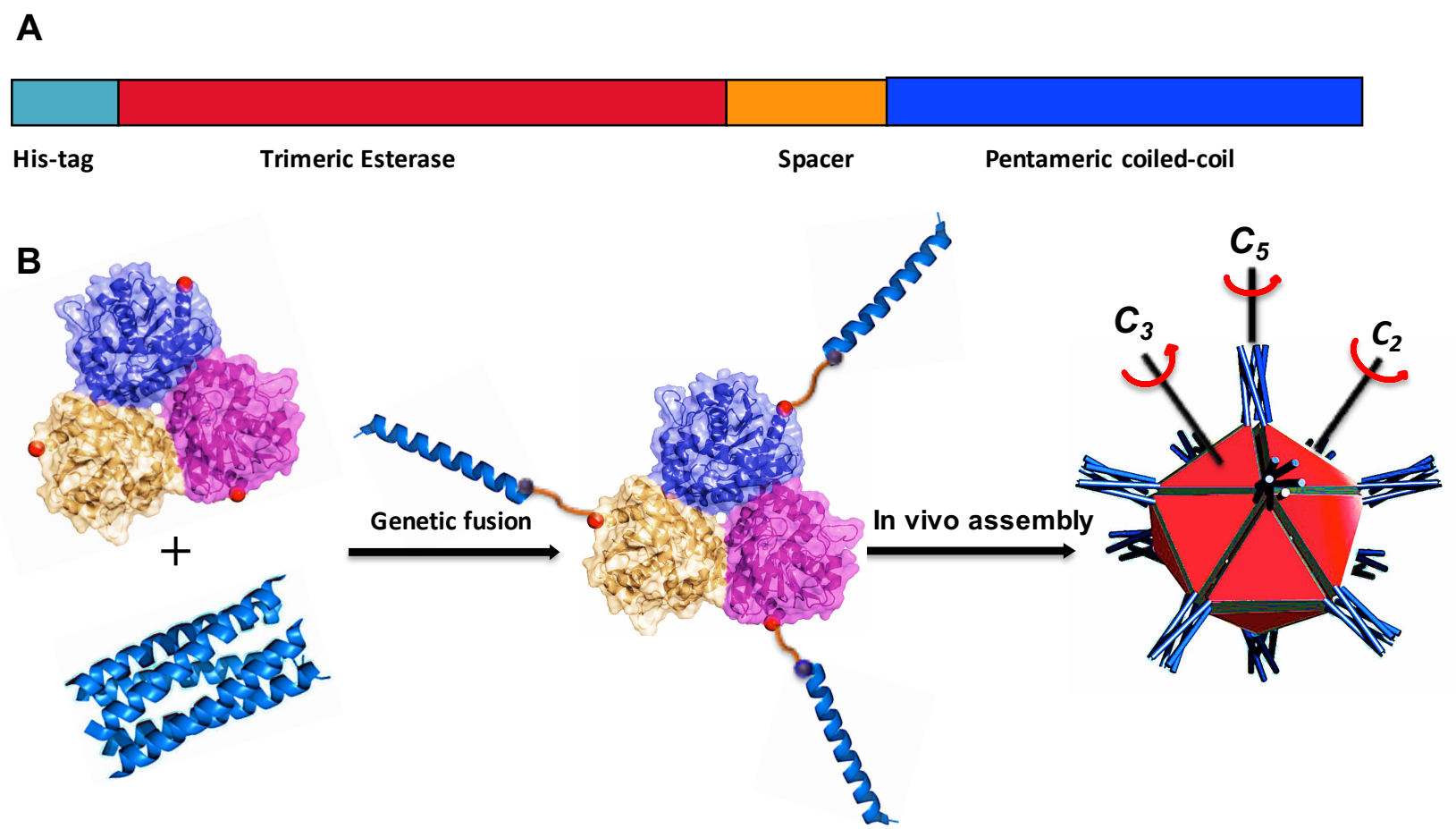

\section{Figure 1}

Design scheme for a self-assembling icosahedral protein cage. A) The Ico8 fusion protein comprises a trimeric esterase an oligo-Gly linker sequence and a pentameric coiled coil. B) Structures of the trimeric esterase and pentameric coiled coil with a schematic illustration of the assembly of the fusion protein into an icosahedral cage. 
A

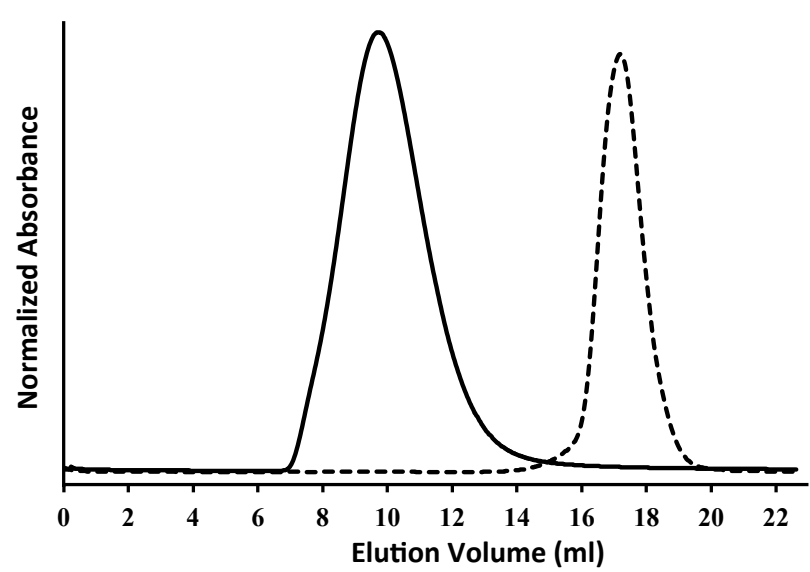

B

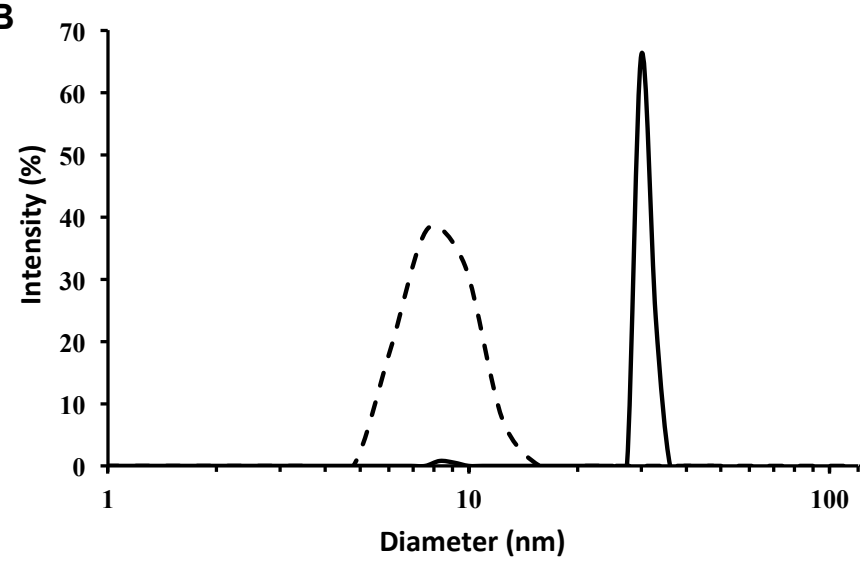

C
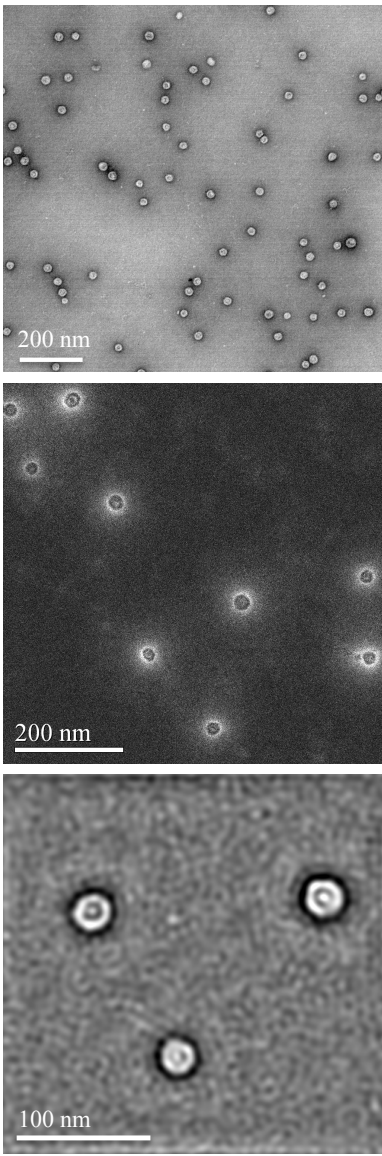

\section{Figure 2}

Characterization of the protein cages formed by Ico8. A) Size and homogeneity of Ico8 assessed by size exclusion chromatography: solid line, Ico8; dashed line TriEst; B) Number-averaged diameter distribution of Ico8 cages measured dynamic light scattering: solid line, Ico8; dashed line TriEst C) Top: Conventional negative stain EM of particles formed by Ico8; middle: highangle annular dark-field scanning EM of Ico8 particles; bottom, bright field Fourier-filtered scanning EM images reveal hexagonal features associated with cages. 

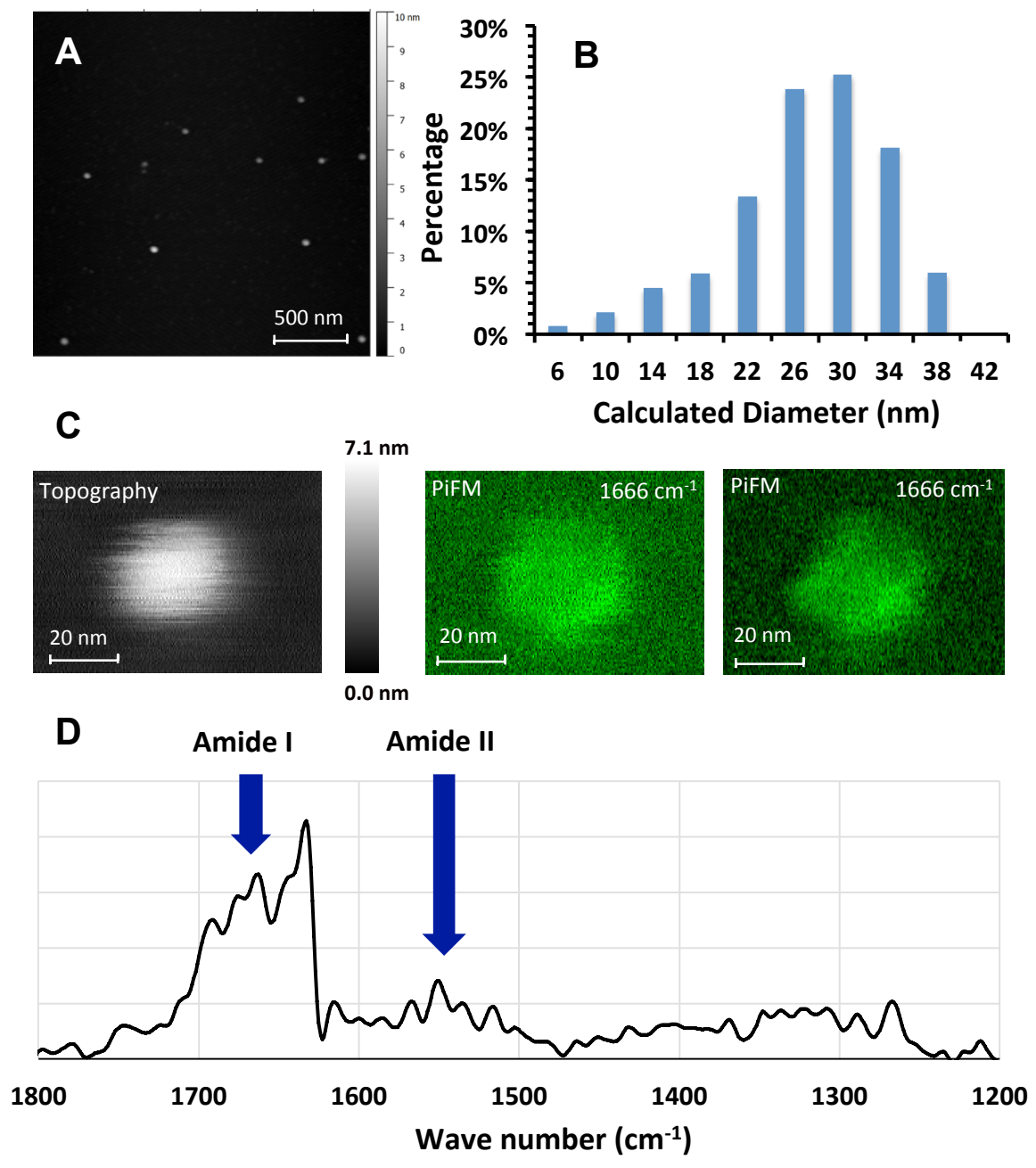

\section{Figure 3}

Atomic force microscopy and IR-photo-induced force microscopy of Ico8 cages. A) Representative field of view of Ico8 particles imaged by AFM. B) Distribution of particle diameters calculated from AFM images. C) left: Topography of a representative Ico8 particle imaged by conventional AFM; middle and right: images of Ico8 particles obtained by scanning PiFM at $1666 \mathrm{~cm}^{-1}$ D) IR spectrum of an individual Ico8 particle. 


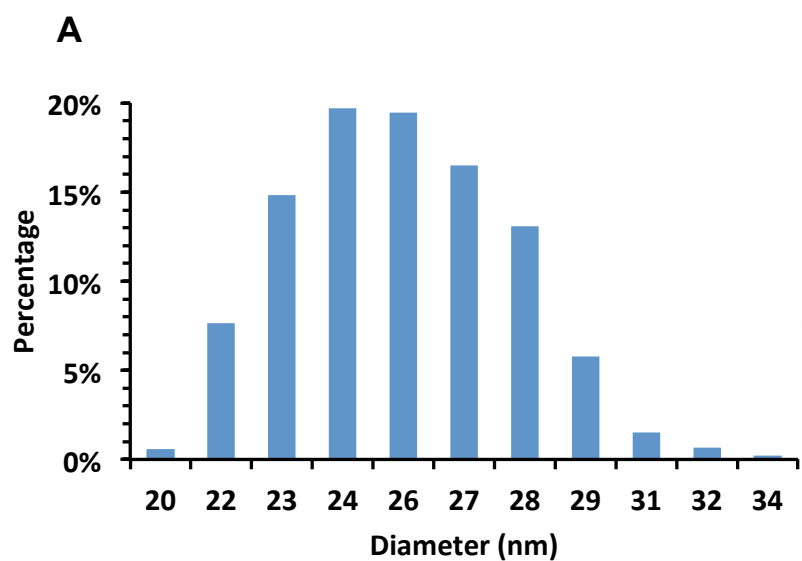

C

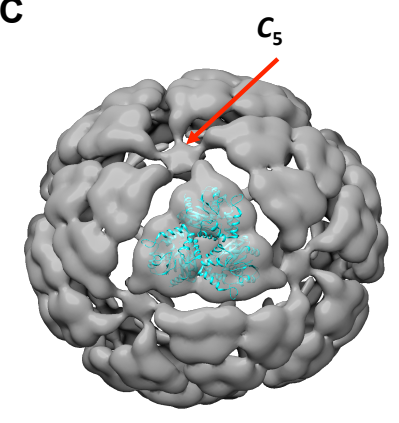

B

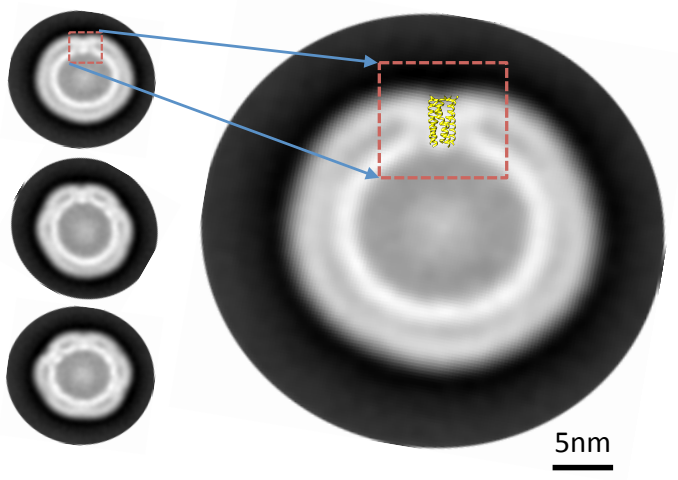

E

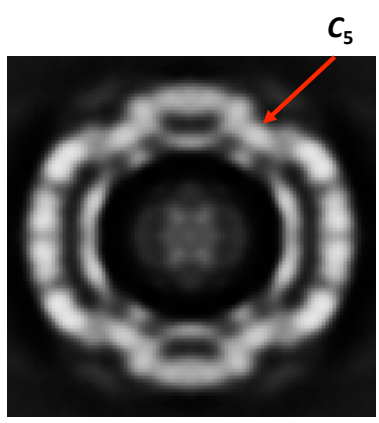

\section{Figure 4}

Cryo EM analysis of Ico8 cages. A) Size distribution of Ico8 cage measured from 2D class averages. B) Representative reference free 2D class averaged images of Ico8 cages illustrating the double-walled appearance of the cages and regularly spaced constrictions between the inner and outer walls that likely represent the coiled coil domain. The expanded view of one class averaged image shows coiled coil domain (yellow) superimposed on the density attributed the coiled coil. C, D) Cross-sections through the 3D electron density map of Ico8, with the crystal structures of TriEst (C) and the pentameric coiled coil (D) docked. E) Cross-section view of density map perpendicular to 2-fold axis. Red arrows indicate the 5-fold symmetry element specified by the coiled coil. 

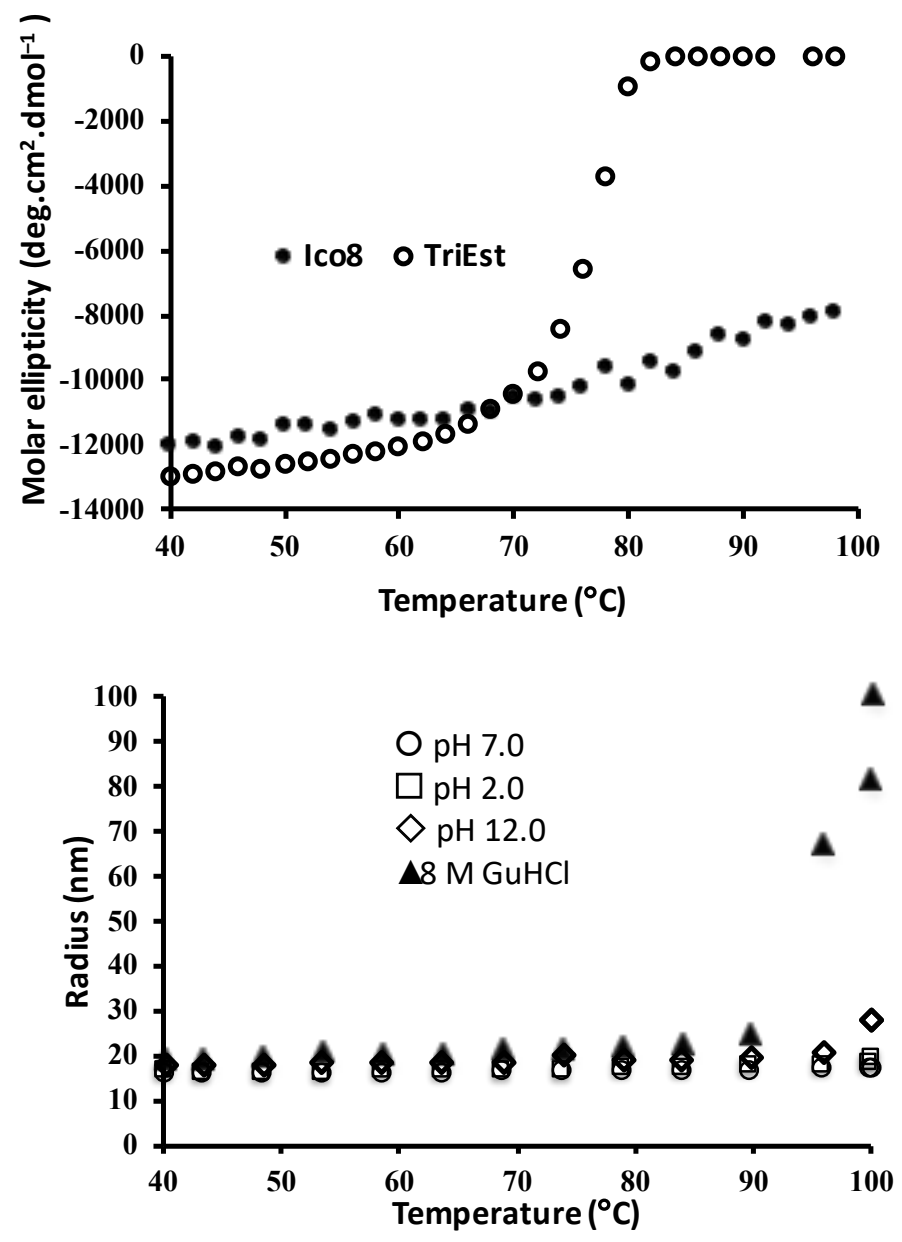

\section{Figure 5}

Thermal and chemical stability of Ico8 protein cage. A) The thermally-induced unfolding of TriEst and Ico8 monitored by changes in molar ellipticity at $222 \mathrm{~nm}$. B) Thermally-induced unfolding of Ico8 monitored by light scattering as a function of temperature at both high and low $\mathrm{pH}$ and in $8 \mathrm{M} \mathrm{GuHCl}$. 


\section{References}

1. Howorka S (2011) Rationally engineering natural protein assemblies in nanobiotechnology. Curr. Opin. Biotechnol. 22, 485-491.

2. Butterfield GL, et al. (2017) Evolution of a designed protein assembly encapsulating its own RNA genome. Nature 552, 415-418.

3. Hsia Y, et al. (2016) Design of a hyperstable 60-subunit protein icosahedron. Nature 535, 136-139.

4. Fletcher JM, et al. (2013) Self-assembling cages from coiled-coil peptide modules. Science 340, 595-599.

5. Lai Y-T, King NP, \& Yeates TO (2012) Principles for designing ordered protein assemblies. Trends Cell Biol. 22, 653-661.

6. Yeates TO (2017) Geometric Principles for Designing Highly Symmetric SelfAssembling Protein Nanomaterials. Ann. Rev. Biophys. 46, 23-42.

7. Yeates TO, Liu Y, \& Laniado J (2016) The design of symmetric protein nanomaterials comes of age in theory and practice. Curr. Opin. Struct. Biol. 39, 134-143.

8. Sciore A \& Marsh ENG (2017) Symmetry-Directed Design of Protein Cages and Protein Lattices and Their Applications. Macromolecular Protein Complexes: Structure and Function, Subcellular Biochemistry), Vol 83, pp 195-224.

9. Kobayashi N \& Arai R (2017) Design and construction of self-assembling supramolecular protein complexes using artificial and fusion proteins as nanoscale building blocks. Curr. Opin. Biotechnol. 46, 57-65.

10. Padilla JE, Colovos C, \& Yeates TO (2001) Nanohedra: Using symmetry to design self assembling protein cages, layers, crystals, and filaments. Proc. Natl. Acad. Sci. U. S. A. 98, 2217-2221.

11. Bale JB, et al. (2016) Accurate design of megadalton-scale two-component icosahedral protein complexes. Science 353, 389-394.

12. King NP, et al. (2014) Accurate design of co-assembling multi-component protein nanomaterials. Nature 510, 103-107.

13. King NP, et al. (2012) Computational Design of Self-Assembling Protein Nanomaterials with Atomic Level Accuracy. Science 336, 1171-1174. 
14. Lai YT, et al. (2014) Structure of a designed protein cage that self-assembles into a highly porous cube. Nat. Chem. 6, 1065-1071.

15. Lanci CJ, et al. (2012) Computational design of a protein crystal. Proc. Natl. Acad. Sci. U.S. A. 109, 7304-7309.

16. Brodin JD, et al. (2012) Metal-directed, chemically tunable assembly of one-, two- and three-dimensional crystalline protein arrays. Nat. Chem. 4, 375-382.

17. Badieyan S, et al. (2017) Symmetry-Directed Self-Assembly of a Tetrahedral Protein Cage Mediated by de Novo-Designed Coiled Coils. ChemBioChem 18, 1888-1892.

18. Cristie-David AS, et al. (2017) Evaluation of de novo-designed coiled coils as offtheshelf components for protein assembly. Molec. Syst. Design Engin. 2, 140-148.

19. Sciore A, et al. (2016) Flexible, symmetry-directed approach to assembling protein cages. Proc. Natl. Acad. Sci. U. S. A. 113, 8681-8686.

20. Zandi R, Reguera D, Bruinsma RF, Gelbart WM, \& Rudnick J (2004) Origin of icosahedral symmetry in viruses. Proc. Natl. Acad. Sci. U. S. A. 101, 15556-15560.

21. Bauler P, Huber G, Leyh T, \& McCammon JA (2010) Channeling by Proximity: The Catalytic Advantages of Active Site Colocalization Using Brownian Dynamics. J. Phys. Chem. Lett. 1, 1332-1335.

22. Smith ML, et al. (2006) Modified Tobacco mosaic virus particles as scaffolds for display of protein antigens for vaccine applications. Virology 348, 475-488.

23. Ma YJ, Nolte RJM, \& Cornelissen J (2012) Virus-based nanocarriers for drug delivery. Adv. Drug Delivery Rev. 64, 811-825.

24. Elmi F, et al. (2005) Stereoselective esterase from Pseudomonas putida IFO12996 reveals alpha/beta hydrolase folds for D-beta-acetylthioisobutyric acid synthesis. $J$. Bacteriol. 187, 8470-8476.

25. Thomson AR, et al. (2014) Computational design of water-soluble alpha-helical barrels. Science 346, 485-488.

26. Nowak D, et al. (2016) Nanoscale chemical imaging by photoinduced force microscopy. Sci, Adv. 2. 10.1126/sciadv.1501571

27. Rajapaksa I, Uenal K, \& Wickramasinghe HK (2010) Image force microscopy of molecular resonance: A microscope principle. Appl. Phys. Lett. 97. 10.1063/1.3480608 
28. Scheres SH (2012) RELION: implementation of a Bayesian approach to cryo-EM structure determination. J. Struct. Biol. 180, 519-530.

29. Grant T, Rohou A, \& Grigorieff N (2018) cisTEM, user friendly software for singleparticle image processing. eLife 7 10.7554/eLife.35383

30. Carrillo-Tripp M, et al. (2009) VIPERdb(2): an enhanced and web API enabled relational database for structural virology. Nucleic Acids Res. 37, D436-D442.

31. Perlmutter JD \& Hagan MF (2015) Mechanisms of Virus Assembly. Ann. Rev. Phys. Chem. 66, 217-239.

32. Roos WH, Bruinsma R, \& Wuite GJL (2010) Physical virology. Nat. Phys. 6, 733-743.

33. Roos WH, et al. (2012) Mechanics of bacteriophage maturation. Proc. Natl. Acad. Sci. U. S. A. 109, 2342-2347.

34. Mastronarde DN (2005) Automated electron microscope tomography using robust prediction of specimen movements. J. Struct. Biol. 152, 36-51.

35. Zheng SQ, et al. (2017) MotionCor2: anisotropic correction of beam-induced motion for improved cryo-electron microscopy. Nat. Methods 14, 331-332. 